\title{
La création d'un lexique interlangues, contraintes et libertés
}

Jean-Paul Barbiche

\section{(2) OpenEdition}

Journals

Édition électronique

URL : http://journals.openedition.org/asp/4337

DOI : 10.4000/asp.4337

ISSN : 2108-6354

Éditeur

Groupe d'étude et de recherche en anglais de spécialité

\section{Édition imprimée}

Date de publication : 1 octobre 1993

Pagination : 395-401

ISSN : 1246-8185

\section{Référence électronique}

Jean-Paul Barbiche, "La création d'un lexique interlangues, contraintes et libertés », ASp [En ligne],

2 | 1993, mis en ligne le 07 mars 2014, consulté le 02 mai 2019. URL : http://journals.openedition.org/ asp/4337 ; DOl : 10.4000/asp.4337

Ce document a été généré automatiquement le 2 mai 2019.

Tous droits réservés 


\title{
La création d'un lexique interlangues, contraintes et libertés
}

\author{
Jean-Paul Barbiche
}

1 J'ai déjà eu l'occasion lors d'un précédent Congrès de vous présenter le travail terminologique effectué à l'Université du Havre dans le cadre du CERTIL (Centre d'Études et de Recherches Terminologiques InterLangues). Je vais aujourd'hui développer mon propos et faire le point par rapport à la dernière fois.

\section{Les origines du projet}

2 L'Université du Havre a été inaugurée en 1986. C'est la plus jeune de France. Elle se compose de deux facultés : l'une est la Faculté des sciences et on y fait malheureusement peu de langues; l'autre est la Faculté des affaires internationales, qui était en réalité, à l'origine, département de l'Université de Rouen et qui est en train de se diversifier vers le droit, les sciences humaines, et l'anglais.

3 Dès l'origine, la volonté d'en faire une faculté ouverte sur l'extérieur a présidé, en particulier par l'enseignement d'une grande variété de langues: toutes les langues d'Europe occidentale cohabitent; plusieurs langues orientales telles que le russe, japonais, chinois, coréen. Des créations sont prévues : malais, vietnamien, thaï, etc. Il y a aussi l'arabe. J'en oublie peut-être.

4 Les filières MST marquent aussi l'ouverture de notre Faculté sur l'extérieur : commerce extérieur, finances internationales, transports internationaux, commerce avec l'Asie, droit international (droit-langue pour être précis), etc. De cet ensemble de caractéristiques, sont nées un certain nombre de nécessités.

5 Aux étudiants de $4^{\mathrm{e}}$ année (diplôme MST) il est demandé de rédiger un mémoire de Terminologie : à partir d'articles de la presse spécialisée, une sélection d'une douzaine de mots clés doit être décortiquée, expliquée, analysée, traduite. C'est un travail minutieux, 
le seul vrai travail de « recherche » intellectuelle qui leur est demandé sur l'ensemble de leur formation.

6 Parallèlement dans le cadre de leur enseignement, dans les filières à débouchés professionnalisés, de nombreux professeurs, chacun dans sa langue, ont ressenti le besoin de rédiger des glossaires ou lexiques bilingues.

7 Toutes ces données ont convergé vers l'idée de créer le Centre d'Études et de Recherches Terminologiques InterLangues, sous mon égide, et d'unir nos efforts dispersés.

8 Nous avons donc décidé de rédiger un lexique multilingue des transports. D'autres suivront. Nous avons aussi des Cahiers, dont je vous parlerai tout à l'heure.

\section{Contraintes}

9 Le thème de ce colloque étant "contraintes et libertés", je vais commencer par les contraintes. Elles sont de toutes natures. L'interlangue, c'est-à-dire l'interdisciplinaire, n'a pas la cote. L'Université aime le traditionnel, se méfie des écarts, et plus encore de l'éparpillement, pour ne pas dire du papillonnement. Elle a sans doute raison dans son principe. Mais là, nous cherchons à enrichir l'Université, pas à la désosser. Car à l'heure de l'Europe unifiée, des ambitions mondialistes, de la communication, il ne suffit plus de traverser une seule frontière et d'en retirer quelques satisfactions personnelles. Il faut voir large, balayer d'un même faisceau plusieurs cultures simultanément (elles-mêmes de nature parfois hybride), concevoir des synthèses d'idées, dialoguer sur plusieurs registres en même temps. Car bien entendu, l'interlangue, c'est aussi de l'interculturel.

Qui dit interdisciplinaire, dit multiplicité des approches intellectuelles, des méthodes de travail, des emplois du temps. Il est très difficile de réunir tous les collègues concernés par un même projet. D'autant plus que Le Havre, plus que tout autre université, souffre du syndrome des transports : beaucoup habitent à Rouen, d'autres à Paris.

11 Les contraintes techniques sont nombreuses, en particulier celle de l'informatique. Chacun d'entre nous dispose d'un matériel personnel différent, incompatible avec celui des autres. Le personnel IATOS est insuffisant, surchargé. Le CERTIL n'a pas de permanent, sauf une CES au service de deux autres centres.

Il a fallu déterminer une méthode de travail propre: nous sommes pour l'essentiel linguistes de formation, pas terminologues ; nous sommes tous des autodidactes (inégaux en qualité) pour ce qui est de l'informatique.

Nous faisons vite le tour de notre potentiel humain. Notre faculté est trop jeune, donc trop petite, nous n'avons pas suffisamment d'enseignants-chercheurs dans nos murs (vacataires, heures complémentaires, professionnels, professeurs associés et postes du secondaire sont légion). Nous sommes aussi surchargés de tâches en tous genres. Nous sommes donc lents, ce qui signifie sans doute inefficaces pour partie. Nous devons trouver des collaborateurs extérieurs. C'est difficile.

L'incompatibilité informatique dont j'ai parlé plus haut apporte des blocages. Le cyrillique entre dans le logiciel, mais ne va pas jusqu'à l'imprimante. Comme nous avons des projets sur le chinois, le japonais et le coréen, j'ai la hantise de ce qui nous attend. 


\section{Les libertés}

15 De quelles libertés bénéficions-nous? Déjà celle de débuter dans un créneau peu revendiqué, et occupé, lorsqu'il l'est, de façon souvent peu satisfaisante. Les ouvrages multilingues sont incomplets, complexes à manipuler, volumineux, trop pointus, etc.

Nous trouvons un débouché pour notre propre travail. Les langues de spécialités sortent de la pénombre grâce aux initiatives de collègues comme Michel Perrin, mais nous sommes loin de bénéficier des structures de recherche et de publications adéquates, en règle générale.

Nous avons sur Le Havre une "clientèle » toute faite pour un glossaire multilingue des transports : nos collègues, nos étudiants, les professionnels des transports. Car vous le savez comme moi, un Centre de recherche de nos jours doit chercher à s'auto-financer, dans toute la mesure du possible. Malheureusement, nous autres littéraires sommes, de ce point de vue, assez peu performants, par rapport aux laboratoires scientifiques.

L'informatique, que j'ai quelque peu critiquée plus haut, essentiellement en raison de l'incompatibilité des différentes marques, présente néanmoins un intérêt essentiel : nous pouvons obtenir une classification à notre goût de façon quasi automatique : on met par ordre alphabétique d'une langue, ou d'une autre, on regroupe, on dissocie, on aligne ou on met en colonnes ( 2,3 ou 4 selon les nécessités), on choisit des caractères comme ci, ou comme ça, on indique le genre avant, ou après le mot, à convenance. Et on peut faire par la suite des études comparatives.

\section{Comment travaillons nous?}

19 Nous avons beaucoup tergiversé ne serait-ce que parce que les premières personnes qui ont travaillé au CERTIL avaient déjà chacune plus qu'une idée: une pratique, une base informatique propre, un travail réalisé. Nous avons d'abord cherché un thème de réalisation. C'est une collègue de portugais qui a proposé sa propre liste de mots en vocabulaire des transports. Ça tombait bien puisqu'il y avait un travail avancé également en anglais, et en allemand. Nous avons en outre sur place des professionnels, mais également des universitaires, linguistes ou non qui sont spécialistes des transports.

Pour la présentation, la méthode «colonne" a ses avantages, mais elle a un gros inconvénient, en particulier dans le multilingue, d'autant qu'à notre niveau nous avons de grandes ambitions : c'est la place. Mille mots traduits en cinq langues, par exemple, cela représente en réalité 6000 mots reproduits cinq fois; ce qui aboutit à des volumes énormes, monopolise les photocopieuses, produit des reliures à la tenue incertaine, etc.

Nous avons donc finalement opté pour une numérotation mixte intra alphabétique et inter alphabétique à partir du français, ce qui va permettre de ne faire qu'une seule liste interlangue: sous chaque nom français apparaîtront les traductions dans toutes les langues où nous avons travaillé. Puis dans chacune des langues étrangères, suivront des index alphabétiques, chaque mot se voyant attribuer le chiffre correspondant sur la liste de référence en langue française. Sur une base de mille mots et cinq langues, nous gagnerons ainsi approximativement un espace équivalent à 25000 mots. Nous en sommes à ce stade d'élaboration. 


\section{de langues que nous le souhaitons, soit nous concentrer sur une langue ou sur un groupe} de langues.

\section{Quel est notre matériau de base?}

Essentiellement, bien entendu, les textes que nous donnons à nos étudiants, ceux que nous leur faisons traduire, ceux qui servent à leurs mémoires de terminologie.

Mais il convient aussi de surveiller l'évolution et la création d'une terminologie sans cesse renouvelée. Nous utilisons donc des revues de professionnels, qui existent dans toutes les langues du monde, y compris l'ukrainien, le croate ou le tagalog lorsqu'il s'agit des organes de syndicats de marins, en anglais parfois allemand, pour les revues d'entreprises; nous recevons aussi les publications des Ports autonomes, etc. C'est un travail qui peut devenir très minutieux, très lourd, voire conduire dans des impasses car l'anglais dominant tout, il est parfois difficile de savoir à quoi correspond tel terme de professionnel en langue espagnole ou coréenne, car la plupart du temps il n'est jamais utilisé. Je ne vous le cacherai pas, ce que nous cherchons à faire est un ouvrage simple, sobre, efficace, maniable, rapide, commercial en un mot.

Mais cette façon de procéder n'est pas nécessairement applicable à toute forme du projet. Dans les transports, le problème est relativement simplifié : un bateau reste un bateau, et un container est un concept assez simple et commun. Je n'en dirai pas tant des formulaires en douane, ou de la notion de RO-RO (Roll on - Roll off) qui peuvent nécessiter qu'on les décrive. Toutefois, les professionnels dans le monde entier se comprennent entre eux. Souvent une « traduction » devient une explication dans la langue traduite.

Le problème, en revanche, sera tout autre pour le droit. Nous sommes actuellement en train d'entamer un travail similaire dans le domaine juridique. Et là, tout passe par une explication de chaque mot: car lawyer, solicitor, attorney ne sauraient se rendre par le mot avocat, et les multiples sens de ce dernier sont loin de recouvrir les mêmes vérités du côté d'une frontière et de l'autre.

27 Sans doute faudra-t-il être plus modeste, se concentrer sur deux ou trois langues seulement, trouver une présentation plus adaptée.

\section{Conclusion, le Certil}

28 Je terminerai en développant les autres tâches que le CERTIL s'est assignées. En dehors de ces glossaires/lexiques interlangues, nous avons créé des Cahiers. Ils ont pour but de publier des articles de Terminologie de toutes spécialités, soit en recherche terminologique proprement dite, soit en didactique, soit en anecdotique.

Nous avons fait paraitre pour l'instant un numéro. Il traite de l'ouverture à l'Est : en RDA/ RFA, en URSS/Russie, plus deux articles de didactique et un sur le gallois.

Il nous est très difficile pour l'instant de planifier sur le long terme. Néanmoins, nous avons quelques pré-projets, certains assez avancés: Terminologie scientifique; un numéro juridique ; un glossaire franco-russe de l'oto-rhino-laryngologie ; des articles sur les régionalismes (corse, gaélique, particularisme de l'argot argentin, les mots étrangers 
utilisés en japonais; un numéro sur l'enseignement. C'est pourquoi vous êtes tous des collaborateurs potentiels du CERTIL.

\section{RÉSUMÉS}

La gestion d'un centre de Terminologie interlangues et la création d'un glossaire interlangues des transports.

Running a multilingual terminolgy centre and the creation of a multilingual transport glossary.

INDEX

Mots-clés : glossaire, terminologie

Keywords : glossary, terminology

\section{AUTEUR}

\section{JEAN-PAUL BARBICHE}

Centre d'Études et de Recherches Terminologiques InterLangues (Certil), Université du Havre. barbiche.famille@wanadoo.fr 